\title{
Atomic Metal Contacts on the Semiconductor by CVD
}

\author{
Chien-Hwa Lung, Ching-Fuh Lin, Che-Chen Chang* \\ National Taiwan University, Taipei, Taiwan 10617
}

\begin{abstract}
The continuing advances in miniaturization of semiconductor devices have seriously challenged the contact technology. This work explored the chemical reaction involved in the bottom-up formation of metal contacts using a linear atomic metal string complex for CVD. The adsorption and surface reaction of the complex on GaN were characterized using $x$-ray photoelectron spectroscopy and secondary ion mass spectrometry. The complex may anchor on the substrate surface with the metal string inclined to the surface. A change in bonding configuration of the chromium metal-string complex took place upon increasing complex dose to the substrate.
\end{abstract}

Index Terms - atomic metal wires, bonding chemistry, CVD, nanocontact technology, XPS.

\section{INTRODUCTION}

The contact technology between metals and semiconductors has drawn much attention in the optoelectronic and electronic industries [1]. The electronic devices are useless unless they are able to communicate with the outside world and with one another. The communication is done through metal contacts. As the critical structure of the integrated circuits currently shrinks in the double-digit nanometer regime, the complexity of the chemical issues related to the metal contacts on semiconductors is dramatically increased. In this regime, the signal transport may be dominated by the chemical and physical [2] effects of atomic-size constraints in the active layers and substrates. Since one of the critical issues in realizing high performance electronic devices is to achieve chemical stability with time and temperature, understanding in the atomic detail of the chemical reactions involved in the formation of the metal contacts thus represents a major challenge to the forefront technology of device fabrication.

In the past, the study of the metal-surface interface has been performed either by depositing various metal halides or carbonyls to the substrate or by exposing the intended substrate to the thermal metal source. Major limitations, however, are present for the use of halides or carbonyls as starting materials in fabricating nanoelectronics. Metal halides need high temperature for conversion via the hydrogen reduction to metal films. Thermal decomposition of metal carbonyls may produce metal films of significantly high resistivity [3] because of the film contamination by carbon and oxygen.

A particular problem also arises at the contact of metal films to the compound semiconductor. When a metal is deposited to the surface of a compound semiconductor substrate, a ternary system is formed. As predicted by the phase rule, most ternary systems tend to form three solid phases in equilibrium. That is, the deposited metal may react chemically with the compound semiconductor substrate to form a three-phase system. It will not be in thermodynamic equilibrium with the substrate, unless the metal happens to form a pseudobinary with the compound semiconductor. Such a chemical reaction, however, may cause the substrate to lose part of the compound and result in the generation of defects in the interface. The device performance and lifetime may thus degrade as a function of time.

Some of the problems addressed above may be circumvented when the metal contact is formed bottom-up from linear atomic metal strings on the semiconductor, instead of top-down from the deposited metal film. With the bottom-up approach, the property of the metal contact formed will be determined mainly by the bonding chemistry rendered from individual metal stings at the contact, instead of the production process, the contamination, or the complication due to the formation of a ternary system.

In this study, the atomic-scale metal contact on the compound semiconductor will be formed by chemically depositing various organometallic compounds at low temperatures compatible with advanced nanoelectronic materials or structures. The chemical deposition of organometallic compounds on the semiconductor surface is a well developed method [4-6]. This work distinguishes itself from those being done previously since the inorganic complex of linear atomic metal strings is used in the deposition. The site-specific bonding of the metal-string complex allows not only the strategic design of the nature of the contact, but also the investigation in detail of the bonding chemistry involved in the formation of the contact.

The linear metal-string compound used in this study is a dipyridylamino complex of the trichromium atom string $[7,8]$. The complex has a central linear string comprised of three $\mathrm{Cr}$ atoms and terminated at both ends 
by $\mathrm{Cl}$ atoms. The central metal string is encaged by four dipyridylamino ligands, which wrap around the string helically in a syn-syn configuration 8]. Each ligand contains two terminal pyridyl groups, bridged together by one $\mathrm{N}$ atom. The two pyridyl groups bond separately to the two terminal $\mathrm{Cr}$ atoms of the string and the bridging $\mathrm{N}$ atom bonds to the middle $\mathrm{Cr}$ atom.

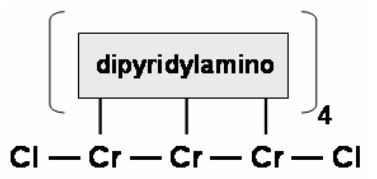

Fig. 1. Schematic of a trinuclear chromium complex. Four 2,2'-dipyridylamino ligands wrap helically around the central linear Cr string helically.

\section{EXPERIMENTAL}

The semiconductor substrate used was a $\mathrm{GaN}(0001)$ film acquired from Cree, Inc. which had a Ga-terminated surface. They were of $4 \mu \mathrm{m}$ thickness grown on sapphire and were undoped, with a carrier concentration of $3.7 \mathrm{x}$ $10^{18} \mathrm{~cm}^{-3}$. Prior to being placed in the analysis system, the samples were subjected to a wet-chemical cleaning process, including treatment of the surface with a $1 \mathrm{M}$ $\mathrm{NaOH}$ solution for $1 \mathrm{~min}$ at room temperature followed by rinse with de-ionized water. After the treatment, the sample was clipped onto a thin tantalum sheet, which in turn was mounted to a XYZ sample manipulator using tantalum clamps. A pair of Chromel-Alumel thermocouples was spot-welded to the tantalum sheet for monitoring the sample temperature.

Once placed in the analysis system, the GaN sample surface was sputtered by high-energy ions to remove impurities on the surface. The analysis system was also equipped with a CLAM II electron energy analyzer, used for x-ray photoelectron spectroscopy (XPS) studies, a quadrupole mass spectrometer, used for static secondary ion mass spectrometry (SIMS), and a retarding field energy analyzer for low-energy electron diffraction (LEED) and Auger electron spectroscopy (AES) studies. The details of the system design were described elsewhere [9]. To inhibit the formation of metallic gallium [10] on the GaN surface during sample cleaning, the sputtering was performed using nitrogen ions. A well-ordered but facetted surface was obtained, as demonstrated by a hexagonal 1x1 LEED pattern with satellite spots [11], after sample cleaning. No oxygen and carbon contamination could be detected by AES.

Molecular evaporation of the trichromium complex was verified by depositing in vacuum a thick film of the complex on a stainless steel plate. Subsequent TGA and
FAB measurements showed identical spectra to those taken before evaporation. The exposure pressure was measured with an ionization gauge and was kept below $\sim 2$ x $10^{-9}$ torr. The dose reported here is the corresponding background exposure at the sample surface and expressed on the unit of langmuir $\left(1 \mathrm{~L}=1 \times 10^{-6}\right.$ torr-s $)$.

XPS studies were performed at $6 \mathrm{~m}$ LSGM beamline of National Synchrotron Radiation Research Center, Taiwan, with the photon energy of $250 \mathrm{eV}$. The incident angle of photons to the surface normal was $45^{\circ}$, and photoelectrons were collected with the analyzer normal to the sample surface. The collected spectra were numerically fitted with the Gaussian function, after Shirley background subtraction. The kinetic energies in the spectra are referred to the binding energy of the metallic $\mathrm{Ga} 3 \mathrm{~d}_{5 / 2}$ at $18.9 \mathrm{eV}$ [12]. SIMS measurements were performed with the sample in line-of-sight of the mass spectrometer. A primary beam of Ar ions of 2-keV energy was used to bombard the sample, with the impact angle measured from the surface fixed at 45 degrees. During the measurement, the ion flux was held in the $0.5-1.5 \times 10^{-9} \mathrm{Amp} / \mathrm{cm}^{2}$ range.

\section{RESULTS AND DISCUSSION}

The reaction and the bonding of the metals at the contact with the semiconductor substrate can be investigated by examining the binding energies of their inner-shell electrons. In general, metals chemically bound to different elements or to different numbers of the same elements possess electronic configurations with the electrons outside their atomic nuclei residing at orbitals of different energy levels. Thus, the electrons ejecting from the inner-shell energy levels in XPS due to the perturbation of the incident $\mathrm{x}$-ray will have different kinetic energies depending on the exact chemical environment the metal atoms have. By measuring the kinetic energy distribution of the inner-shell electrons emitting from the sample surface, one may then determine the chemical nature of the metals formed at the contact.

The chemistry involved in the reaction of $\mathrm{GaN}$ with the inorganic complex of the linear trichromium string was thus first studied using XPS. Shown in Fig. 2 are the kinetic energy distribution of the electrons ejecting from the $\mathrm{Cr} 3 \mathrm{p}$ orbital of the sample surface exposed at $110 \mathrm{~K}$ to the trichromium complex of the indicated doses. A broad XPS profile, with the full width at half maximum of $\sim 2.5 \mathrm{eV}$, was obtained as the sample surface was exposed to the complex of $0.80 \mathrm{~L}$. Its intensity increased with the complex exposure. The fact that the XPS profile obtained from the sample, which was not a metallic substrate, was not quite symmetric indicated that there was more than one chemical state associated with the $\mathrm{Cr}$ atoms present in 
the complex-exposed $\mathrm{GaN}$ system. Inspecting the molecular structure of the trichromium complex shows that a free complex molecule should thus possess two different $\mathrm{Cr}$ chemical states.

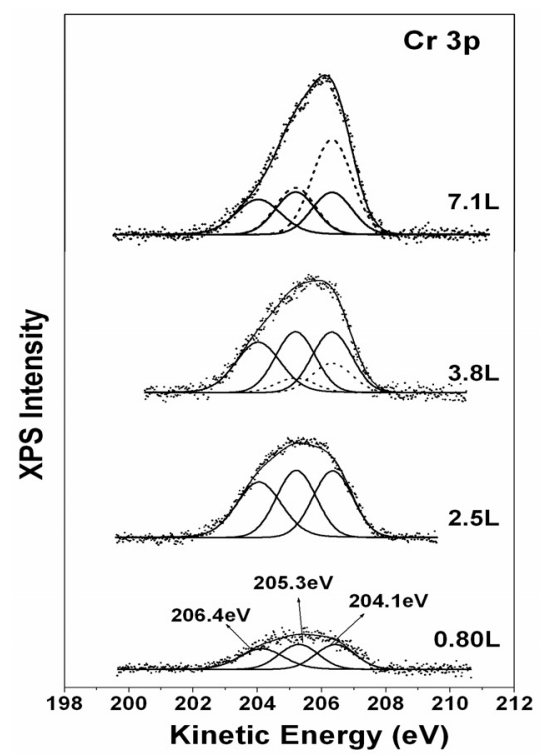

Fig. 2. XPS spectra of $\mathrm{Cr} 3 p$ obtained from the GaN surface exposed at $110 \mathrm{~K}$ to the indicated doses of trichromium complex.

In order to extract chemical state information displayed in Fig. 2, deconvolution of the $\mathrm{Cr} 3 \mathrm{p}$ XPS profiles was carried out by peak fitting. It showed that the $\mathrm{Cr} 3 p$ spectra obtained at exposures of less than $\sim 3.0 \mathrm{~L}$ could not be fitted well with only two component peaks each. The best fit of these spectra was obtained when at least three subpeaks, positioned at $204.1 \mathrm{eV}, 205.3 \mathrm{eV}$, and 206.4 $\mathrm{eV}$, respectively, were assumed in the fitting. There were thus three chemical states associated with the $\mathrm{Cr}$ atoms present on the sample surface in the low exposure regime of less than $\sim 3.0 \mathrm{~L}$. It indicated that the reactions of the complex with GaN led to $\mathrm{Cr}$ bonding chemically to the surface and brought about a new chemical state for the bonding $\mathrm{Cr}$ atom, which was in a chemical environment different to those of the unreacted $\mathrm{Cr}$ atoms in the complex.

To confirm the Cr chemical bonding to the GaN surface, the XPS spectra of the electrons ejecting from the $\mathrm{Cl} 2 \mathrm{p}$ orbital of the sample surface were measured. As shown in Fig. 3, the $\mathrm{Cl} 2 \mathrm{p}$ XPS peak centered at $\sim 51.2 \mathrm{eV}$ corresponds to $\mathrm{Cl} 2 \mathrm{p}_{3 / 2}$ and the one at $\sim 49.6 \mathrm{eV}$ to $\mathrm{Cl} 2 \mathrm{p}_{1 / 2}$. These spin-orbit peaks were separated by about $1.60 \mathrm{eV}$, in good agreement with the spin-orbit splitting value of $\mathrm{Cl}$ $2 \mathrm{p}$. The $\mathrm{Cl} 2 \mathrm{p}_{3 / 2}$ peak obtained at exposures of less then $3.0 \mathrm{~L}$ can be fitted with two component peaks (solid curves) at binding energies of $50.0 \mathrm{eV}$ and $51.1 \mathrm{eV}$, respectively, and the $\mathrm{Cl} 2 \mathrm{p}_{1 / 2}$ peak fitted with two component peaks (dash curves) at $48.4 \mathrm{eV}$ and $49.5 \mathrm{eV}$, respectively. Because of the identical bonding configuration of the two $\mathrm{Cl}$ atoms in the complex molecule, the observations of two features in the $\mathrm{Cl} 2 \mathrm{p}_{3 / 2}$ spectrum and the dose-independent ratio of the peak areas indicated that in the low exposure region, the complex decomposed on the GaN surface with one of the two terminal $\mathrm{Cr}-\mathrm{Cl}$ bonds of the complex disrupted to yield two different $\mathrm{Cl}$ chemical states.

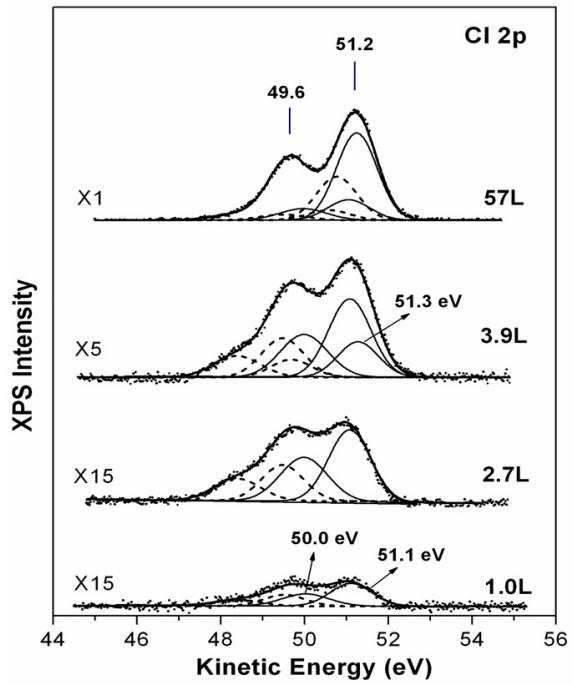

Fig. 3. XPS spectra of $\mathrm{Cl} 2 \mathrm{p}$ obtained from the GaN surface exposed at $110 \mathrm{~K}$ to the indicated doses of trichromium complex.

For the complex to chemisorb on the sample surface with its $\mathrm{Cr}$ atom string oriented parallel to the surface, the two terminal $\mathrm{Cr}$ atoms of the string should have given rise to XPS signals at the same binding energy, resulting in a Cr $3 p$ XPS profile comprising of only two component peaks. Three subpeaks were observed, however, in the $\mathrm{Cr}$ 3 XPS spectra (Fig. 2) measured at low exposures of less than $\sim 3.0 \mathrm{~L}$. In addition, the peak areas of the three $\mathrm{Cr}$ subpeaks were about equal in each of the spectra obtained at low exposures. It revealed that for each adsorbed complex, only one of the two terminal $\mathrm{Cr}-\mathrm{Cl}$ bonds in the trichromium complex was broken upon adsorption and that all the adsorbed complex anchored to the sample surface with one end of the string bonding to the surface and the other remaining free. Furthermore, increasing the substrate temperature to $300 \mathrm{~K}$ did not alter the XPS spectra of $\mathrm{Cr} 3 \mathrm{p}$ and $\mathrm{Cl} 2 \mathrm{p}$ obtained, indicating that the anchored metal string was stable up to room temperature.

The presence of two additional component peaks, with the ratio of the peak areas of $2: 1$, at high doses of more 
than $\sim 3.0 \mathrm{~L}$ indicated that the complexes of high dose may adsorb with their string axis oriented parallel to the surface. With such a bonding configuration, the absence of $\mathrm{Cr}-\mathrm{Cl}$ bond breakage resulted in a single new $\mathrm{Cl} 2 \mathrm{p}$ feature in Fig. 3 observed at $51.3 \mathrm{eV}$ at $>3.0 \mathrm{~L}$.

The extent the trichromium complex decomposes on the sample surface may be explored by studying the SIMS spectra taking from the sample surface. As shown in Fig. 4, all the SIMS peak intensities, except the $\mathrm{Ga}^{+}(\mathrm{m} / \mathrm{e} 69$, 71) peak intensity, increased as the trichromium complex

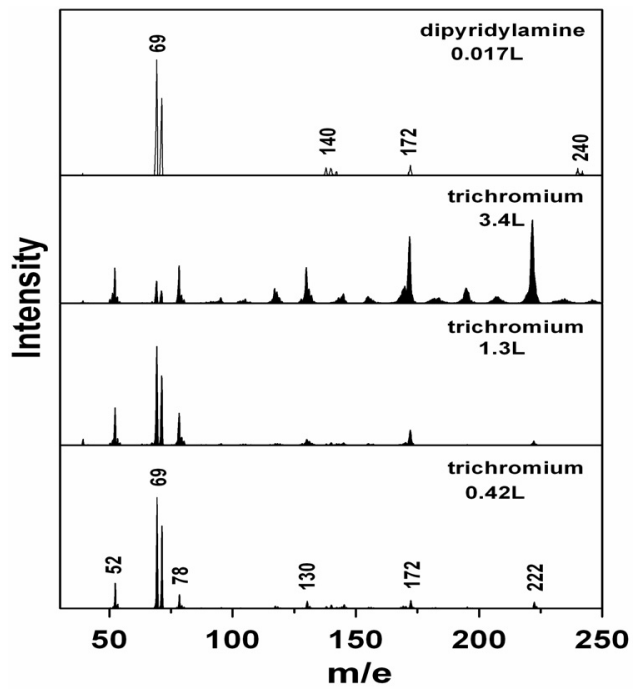

Fig. 4. SIMS spectra of GaN exposed to the indicated doses of the trichromium complex and dipyridylamine, respectively. All spectra are recorded at $105 \mathrm{~K}$.

exposure was increased to 3.0L. SIMS spectra of the model compound of dipyridylamine, which is the ligand molecule of the complex, adsorbed on the sample surface were taken for comparison. As shown in the figure, which was normalized to the $\mathrm{m} / \mathrm{e} 69$ signal intensity, the major peaks obtained included those due to the sputtering of $\mathrm{Ga}_{2}$ $(\mathrm{m} / \mathrm{e} 138,140,142)$, the protonated ligand $(\mathrm{m} / \mathrm{e} 172)$, and the Ga-ligand adduct $(\mathrm{m} / \mathrm{e} 240,242)$. The spectrum contained no signals from the fragments of the ligand molecule, indicating that the ligand exposure to the sample surface resulted in molecular adsorption. The observation of the m/e 240 and 242 peaks revealed that the ligand molecule may adsorb molecularly on the Ga site of the surface. There were no signals detected at these two $\mathrm{m} / \mathrm{e}$ values from the sample surface exposed to the complex, however. It indicated that the complex structure may remain intact upon adsorption at $105 \mathrm{~K}$ on the sample surface. The ligands of the adsorbed complex, however, may be sputtered from the adsorbed molecules during SIMS measurement to give the observed peak at m/e 172 .
The sputter desorption also gave rise to the pyridyl-Cr (m/e 130) and ligand-Cr (m/e 222) adduct peaks observed in the spectrum. The absence of the pyridyl-Ga and ligand-Ga peaks in the spectrum supports the adsorption of the structure-intact complex on GaN. In addition, the change in the SIMS intensity distribution obtained at $>3.0$ $\mathrm{L}$ confirms that a change in the complex adsorption behavior occurs at high doses, as discussed above.

\section{CONCLUSION}

A dipyridylamino-chelated linear trinuclear chromium string complex was employed as a model complex to explore the reaction chemistry involved in the bottom-up formation of metal contacts on the compound semiconductor substrate. The terminal $\mathrm{Cr}-\mathrm{Cl}$ bond of the string was disrupted and the atomic metal string anchored to the $\mathrm{GaN}$ substrate at low doses. It remained structurally intact at room temperature. A chemisorption layer of metal strings is formed on $\mathrm{GaN}$ at $\sim 3.0 \mathrm{~L}$. Further increasing doses caused the metal string complex to adsorb molecularly on top of the chemisorption layer.

\section{ACKNOWLEDGEMENT}

This work was supported by ROC National Science Council (NSC93-2113-M002-003, NSC93-2120-M002011). The authors gratefully thank Prof. Shi-Ming Peng for providing the trichromium complex sample.

\section{REFERENCES}

[1] K. Suguro, H. Shibata, Advanced Metallization for ULSI Applications, eds. D. P. Favreau, Y. Shacham-Diamond, Y. Horiike, San Diego, CA, 1993, (Materias Research Society, Pittsburgh, PA, 1994.)

[2] G. H. Dohler, Scientific American 249, 144, 1983.

[3] Y. Pauleau, Mater. Technol 3 (1989) 47.

[4] W. H. Bowser, W. H. Weinberg, J. Am. Chem. Soc. 103, 1453,1981

[5] M. L. Green, R. A. Levy, J. Mater. 6, 63, 1985.

[6] R. L. van Hemert, L. B. Spendlove, R. E. Sievers, J. Electrochem. Soc. 112, 1123, 1965.

[7] T. B. Tsao, G. H. Lee, C. Y. Yeh, S. M. Peng, J. Chem. Soc.-Dalton Trans. 1465, 2003.

[8] C.-C. Wang, W. -C. Lo, C.-C, Chou, G.-H. Lee, J.-M. Chen, S.-M. Peng, Inorg. Chem. 37, 4059, 1998.

[9] C.-C. Chang, I.-J. Huang, C.-H. Lung, H.-Y. Hwang, L.-Y. Teng, J. Phys. Chem. B105, 994, 2001.

[10] Y. Ould-Metidji, L. Bideux, D. Baca, B. Gruzza, V. Matolin, Appl. Surf. Sci. 212/213, 614, 2003.

[11] V. J. Bellitto, B. D. Thomas, D. D. Koleske, A. E. Wickenden, R. L. Henry, Surf. Sci. 430, 80, 1999.

[12] Y. H. Lai, C. T. Yeh, J. M. Hwang, H. L. Hwang, C. T. Chen, W. H. Hung, J. Phys. Chem. B 105, 10029, 2001. 
